\title{
Effect of Splitters in Recirculation Channels on Performance of Turbocharger Compressors Used in Gasoline Engines- A CFD Study
}

\author{
N. Nithesh ${ }^{1}$ and S. Prajwal ${ }^{2 *}$ \\ ${ }^{1}$ Department of Mechanical Engineering, Manipal Institute of Technology, \\ Manipal Academy of Higher Education, Manipal, Karnataka, India \\ ${ }^{2}$ Department of Mechatronics Engineering, Manipal Institute of Technology, \\ Manipal Academy of Higher Education, Manipal, Karnataka, India \\ *Email: Prajwal.shenoy@manipal.edu \\ Phone: +91 8095782237
}

\begin{abstract}
Turbochargers used in gasoline engines have their compressor outlet directly coupled to the engine inlet via the throttle valve. On sudden closing of the throttle, the compressor outlet is blocked, and the compressed air has no path to exit resulting in a compressor surge. Compressor recirculation valves are used to connect the outlet of the compressor to the inlet to recirculate excess air and thus reduce the compressor surge. Under normal operating conditions, when the valve is closed, the channel connecting the compressor inlet and the valve causes an inlet disturbance resulting in the reduction of compressor efficiency. Hence a steady state CFD analysis of a gasoline engine turbocharger compressor modelled with a recirculation channel at the inlet was conducted. The channel connecting the compressor inlet and the recirculation valve was observed to cause inlet aerodynamic disturbance resulting in a drop in compressor efficiency by $1 \%$. To overcome this problem, splitters were used in recirculation channels and $80 \%$ recovery of loss was observed with the use of splitters.
\end{abstract}

Keywords: Turbomachine, recirculation channel, splitters, CFD

\section{INTRODUCTION}

Compressor Recirculation Valves (CRV's) are usually fitted in a gasoline engine turbocharger compressor to prevent compressor surge. Closing the throttle valve suddenly blocks the compressor outlet resulting in excessive pressure rise in the compressor which shifts the operating region of the compressor to surge. The excess air from the outlet is recirculated back to the inlet with the help of a CRV. But under normal operating conditions, when the CRV is closed, the recirculation channel (RC) connecting the CRV and the inlet pipe was observed to cause aerodynamic disturbances at the inlet resulting in a drop in compressor performance. To reduce the inlet distortion, splitters are modeled in the RC and its effect on the compressor performance is studied. Splitters are modelled and the compressor performance is compared between the following cases viz; Baseline (No RC), without RC (without splitters), and with RC (splitters attached).

The effect of inlet distortion on compressor performance has been previously studied. Experimental analysis of compressor performance due to inlet distortion has been studied using artificially induced disturbances at the inlet [1-4]. Layers of honeycomb structure was placed at the inlet to produce radial and circumferential distortion. A loss in compressor efficiency was observed because of the inlet distortion. Also, it has been 
observed that bent pipe at the inlet of radial machines offer lesser efficiency than compressors with straight inlet pipes [5-6]. Inlet distortion due to pipe bends or other obstructions resulting in change in inlet flow distribution and further resulting in blade failures due to resonance has been reported [7-8]. The impeller upstream flow structure, its mechanism and its effect on the performance of the impeller and the compressor stage has been presented [9-13]. Comparison of numerical and experimental flow field in a bent duct has been demonstrated in [14-18]. No literature is observed which aims to reduce the compressor losses because of the inlet distortions caused by the RC. Hence in this study, the distortion caused by the RC at the inlet is studied using CFD and use of splitters in $\mathrm{RC}$ and its role in recovering losses is discussed.

\section{DESIGN AND ANALYSIS METHODOLOGY}

A figure depicting a compressor fitted with a recirculation valve is shown in Figure 1(a). A recirculation channel and a CFD model of splitter on bent pipe is shown in Figure 1(b) and Figure 1(c), respectively.

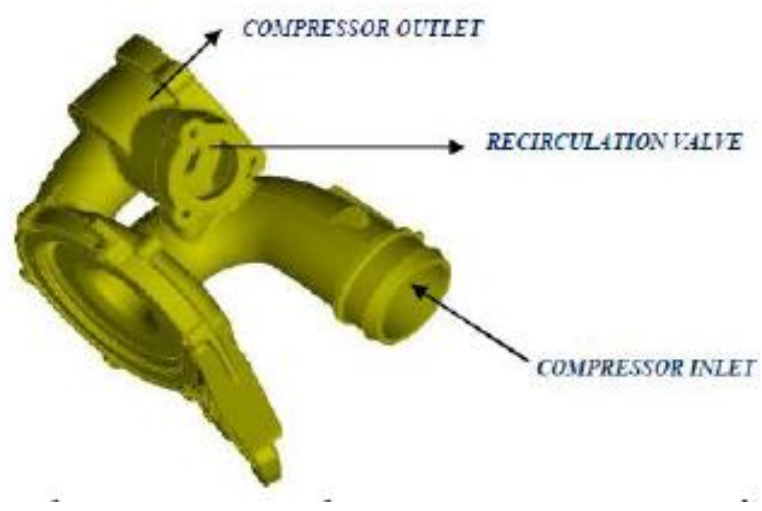

(a)

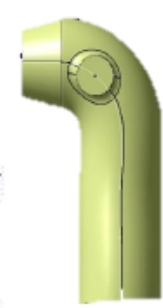

(b)

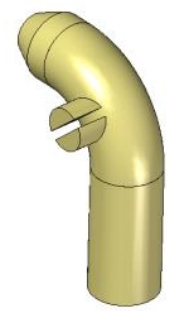

(c)

Figure 1. Details of (a) recirculation valve (b) RC modelled on pipe and; (c) splitter modelled in RC

\section{Modelling and Meshing}

The CFD model consists of a volute, impeller and a bent inlet pipe with the RC modelled on it. The gas path for the volute is extracted, diffuser width is modelled appropriately and meshed using tetrahedral elements using a commercial CFD software. Prism layers are provided to account for boundary layer effects. The extracted and meshed volute casing is shown in Figure 2. The blade is meshed using hexahedral elements. The shroud curve is modified to match the required trim. The meshed blade is shown in Figure 3 . The inlet bent pipe is modeled with a Bend ratio (B.R.) $=0.75$ with bend ratio defined as $r / D$, where $r$ is radius of the bend and $\mathrm{D}$ is diameter of the bent pipe. The inlet pipe along with $\mathrm{RC}$ is meshed using hexahedral elements. All the three domains are then assembled for CFD analysis. The assembled CFD model without any RC is as shown in Figure 4. The $\mathrm{RC}$ of diameter $26 \mathrm{~mm}$ was modelled on the bent pipe. Splitters are modelled in two different configurations, viz. splitter perpendicular to streamline flow and splitter along the streamline flow. The two configurations of splitters are shown in Figure 5. 

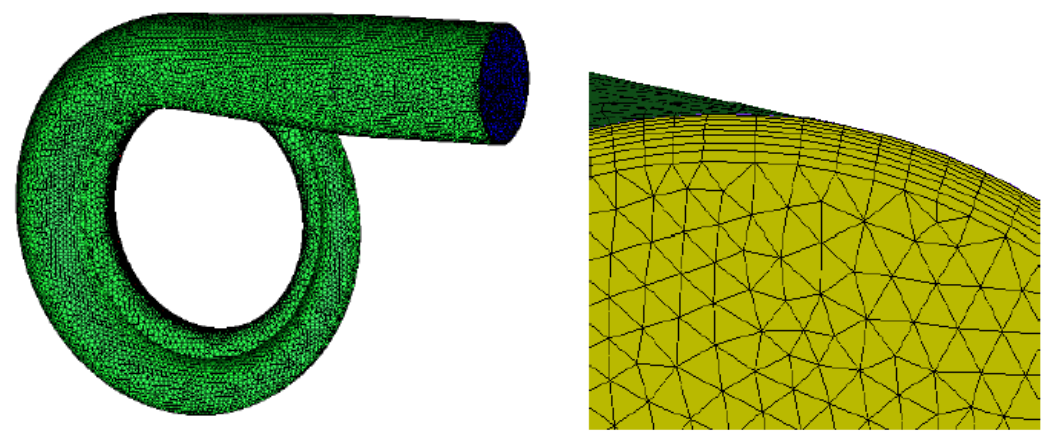

Figure 2. Volute tetrahedral mesh with prism layers.
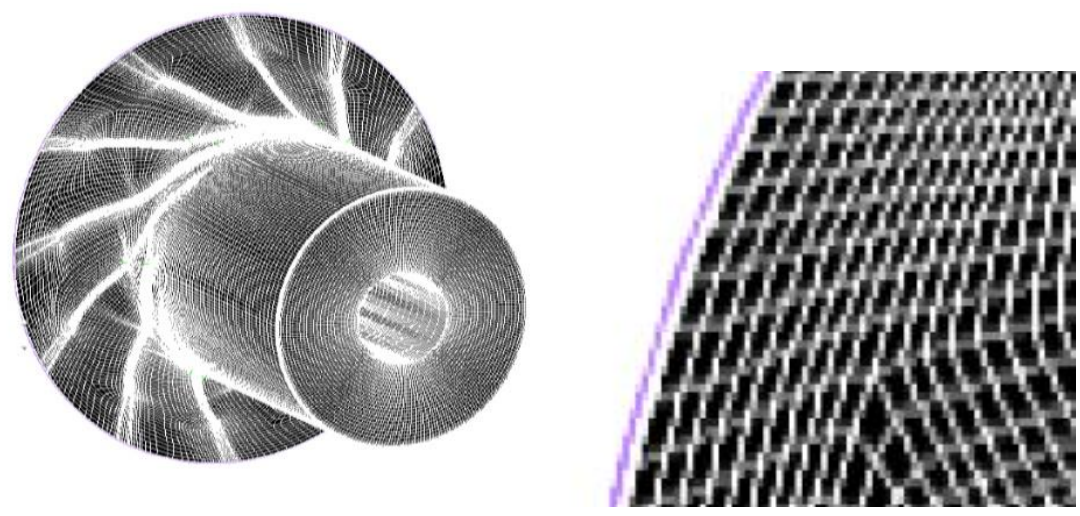

Figure 3. Wheel meshed using hexahedra elements.

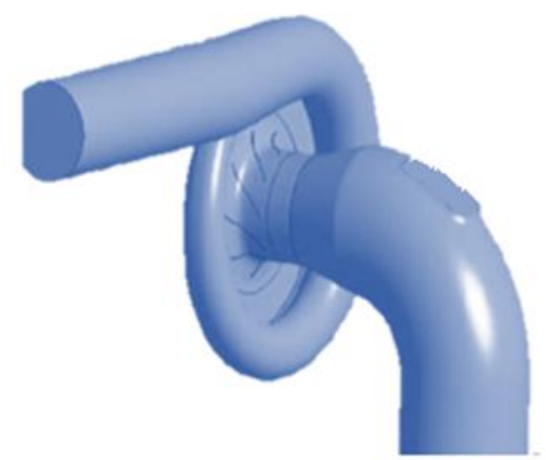

Figure 4. Assembled domain for CFD analysis depicting inlet pipe without RC.
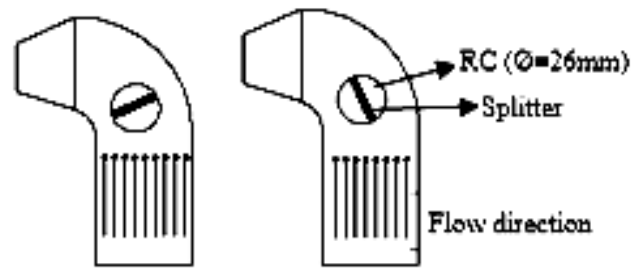

Figure 5. Splitter configurations. 


\section{Boundary Conditions, Solution and Post Processing}

The simulation is carried out for single speed with three operating conditions, viz. surge, peak and choke point. A static pressure boundary condition is provided at the outlet and total quantities of atmospheric pressure and temperature was provided at the inlet. The $\mathrm{RC}$ is treated as a wall on all sides with no slip condition. The rotor is treated as a rotating domain and was provided with an angular velocity boundary condition. Once the solution is obtained, the efficiency of the compressor is calculated. Overall efficiency is calculated using Equation 1. Wheel efficiency is calculated using Equation 2. Graphs of efficiency versus corrected mass flow rate is plotted for different cases. The following cases are used for the study:

i. Analysis of compressor performance without RC

ii. Analysis of compressor performance with $\mathrm{RC}$

iii. Analysis of compressor performance with RC, with splitter along the flow

iv. Analysis of compressor performance with RC, with splitter perpendicular to flow.

$$
\begin{array}{r}
\eta=\frac{P_{2 T} \frac{\gamma-1}{\gamma}-1}{P_{1 T} \frac{\gamma-1}{\gamma}-1} \\
\eta=\frac{\mathrm{P}_{2 \mathrm{WT}} \frac{\gamma-1}{\gamma}-1}{\mathrm{P}_{1 \mathrm{WT}} \frac{\gamma-1}{\gamma}-1}
\end{array}
$$

where $\mathrm{P}_{2 \mathrm{~T}}$ and $\mathrm{P} 2{ }_{\mathrm{WT}}$ are total pressures at exit of compressor and wheel, respectively, $\mathrm{P}_{1 \mathrm{~T}}$ and $\mathrm{P}_{1 \text { WT }}$ are total pressures at inlet of compressor and wheel, respectively, and $\gamma$ is adiabatic index.

\section{VALIDATION OF CFD TOOL USING EXPERIMENTAL RESULTS}

Experiments were carried out by welding the $\mathrm{RC}$ on the outlet region of the compressor housing and the results were compared with numerical solutions to investigate the sensitivity of the numerical simulation in replicating the nature of flow within the RC. The pressure was varied using a valve at the outlet. The loss in efficiency observed in the experimental results are compared with the loss obtained using numerical study. Loss can be defined as the drop-in efficiency (calculated by Eq. (1) by measuring the total pressure at inlet and outlet) of a compressor with the $\mathrm{RC}$ when compared to the efficiency of the same compressor without the RC. The RC was modelled on the outlet at $90^{\circ}$ from $\mathrm{T}-\mathrm{T}$ section as shown in Figure 6(a). The diameter used was $18 \mathrm{~mm}$ and experiments were conducted for two RC heights of $10 \mathrm{~mm}$ and $20 \mathrm{~mm}$. The CFD model of the same is shown in Figure 6(b). 


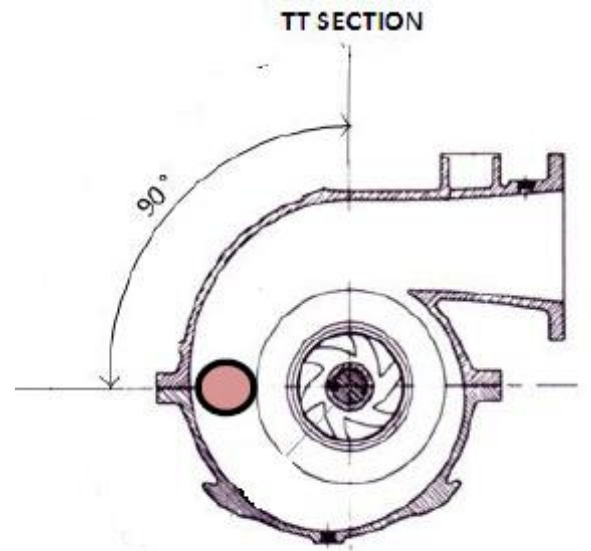

(a)

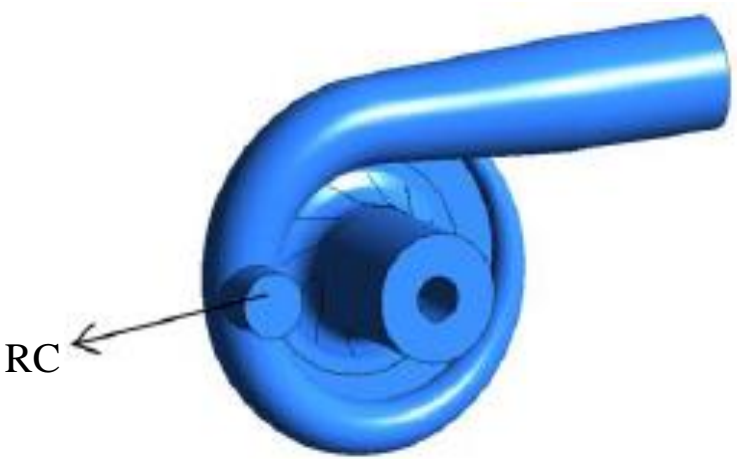

(b)

Figure 6. RC modelled on the housing of the compressor in (a) schematic representation and; (b) CFD model.

The results of experiments and numerical simulation is shown in Figures 7(a) and 7(b) with the values tabulated in Table 1 . CFD results match with close tolerance in case of $\mathrm{RC}$ at $90^{\circ}$ from TT both in $10 \mathrm{~mm}$ as well as $20 \mathrm{~mm}$ cases. It can be observed that in the case of RC of height $10 \mathrm{~mm}$ CFD predicts $0.75 \%$ loss at peak whereas experiment predicts $0.8 \%$ loss and in the case of $20 \mathrm{~mm}$ high RC CFD predicts $1.5 \%$ loss at peak whereas experiment predicts $1.2 \%$ loss.

Table 1. Comparison of percentage loss at surge, peak and choke points

\begin{tabular}{ccccccc}
\hline \multirow{2}{*}{ Height of RC } & \multicolumn{2}{c}{ \%Loss at Surge } & \multicolumn{2}{c}{ \%Loss at Peak } & \multicolumn{2}{c}{ \%oss at choke } \\
\cline { 2 - 7 } & CFD & EXP & CFD & EXP & CFD & EXP \\
\hline $10 \mathrm{~mm}$ & 0.61 & 0.9 & 0.75 & 0.8 & 0.97 & 1.1 \\
$20 \mathrm{~mm}$ & 1.05 & 0.9 & 1.5 & 1.2 & 1.3 & 1 \\
\hline
\end{tabular}

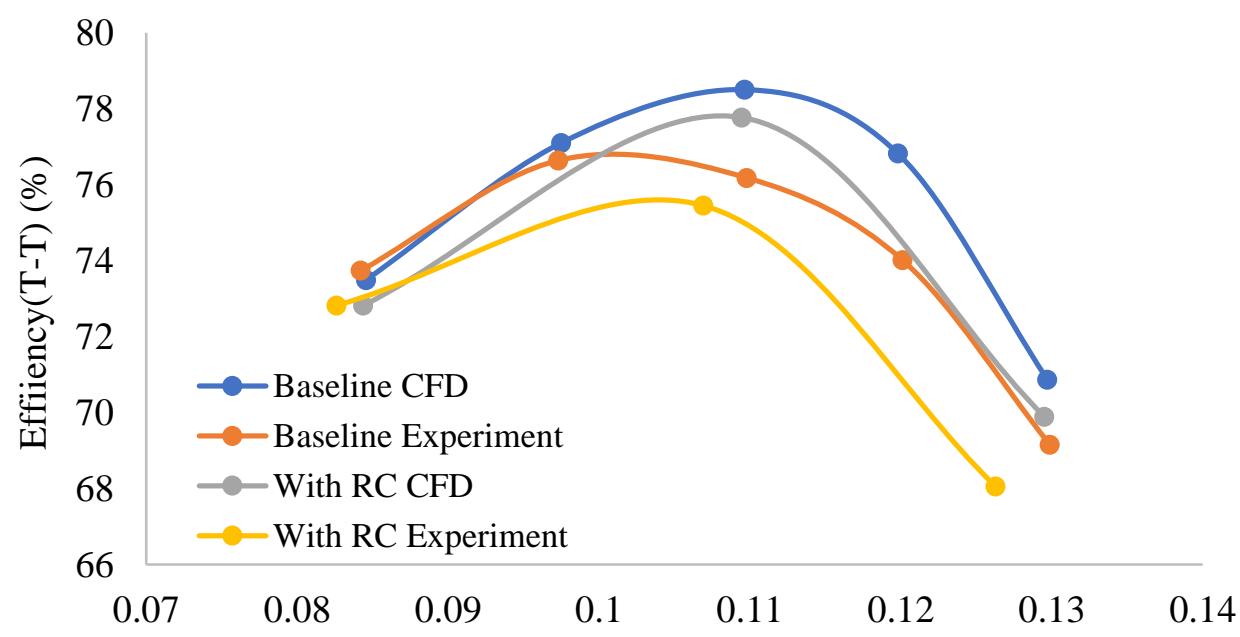

Corrected Mass Flow Rate (kg/s)

(a) 


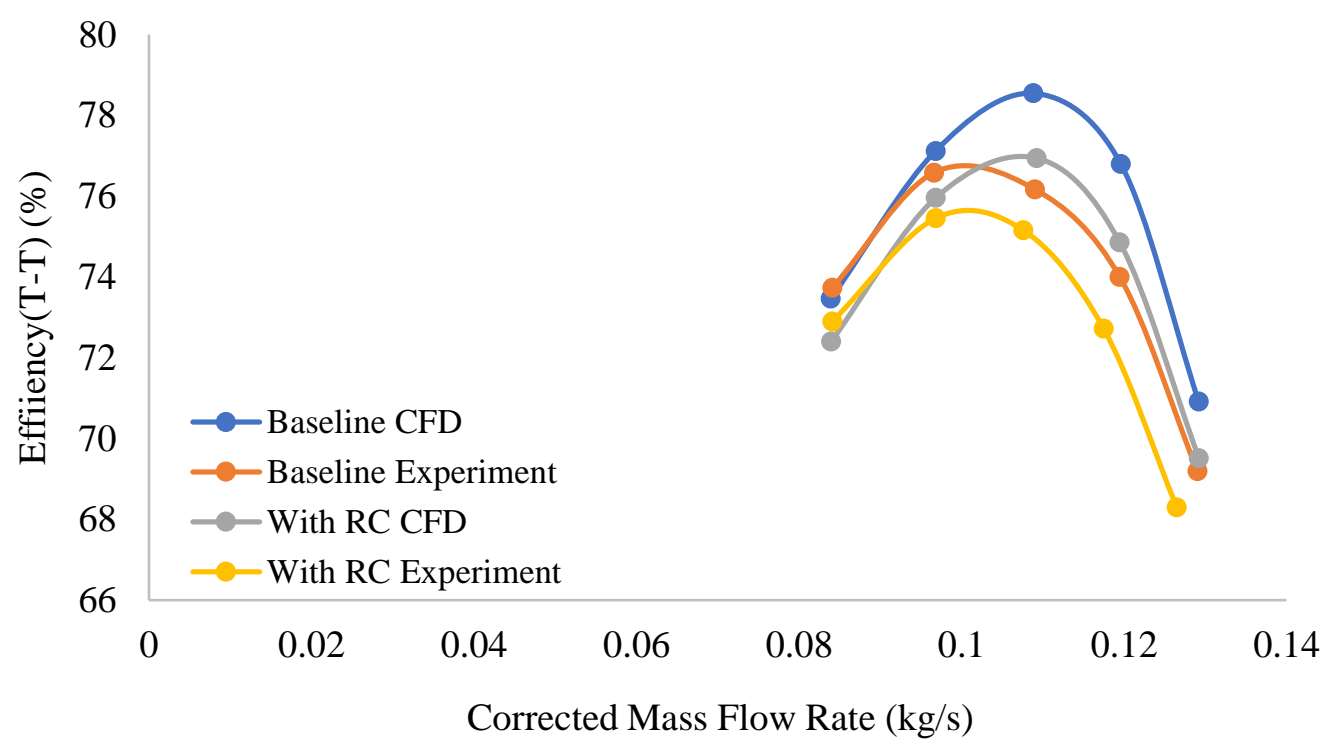

(b)

Figure 7. Comparison of efficiency curves of experimental and numerical results for (a) $10 \mathrm{~mm}$ and (b) $20 \mathrm{~mm}$ height

Evolution of flow at 5 different planes is shown in Figure 8 where velocity vector plots in 5 different planes are depicted. It can be observed from Figure 8 that in the case of $20 \mathrm{~mm} \mathrm{RC}$, the vortex formed in the RC interacts with the primary flow causing distortion in the primary flow which results in loss of compressor efficiency. It can be observed that CFD results match well with the experimental results and the mesh size, and the boundary conditions will be used for further study. Even though the comparison of experimental and CFD results was done using an RC modelled at the outlet, it is assumed that a similar effect is captured by CFD in the RC modelled on the inlet pipe.

\section{RESULTS AND DISCUSSION}

The test matrix is shown in Table 2. The compressor performance was first evaluated without any RC modelled on it (baseline). All other cases were compared with the baseline.

Table 2. Test matrix

\begin{tabular}{lcc}
\hline Case & Maximum Loss & Loss Recovery \\
\hline Baseline (No RC) & NA & NA \\
RC without splitter & $1 \%$ & NA \\
RC with splitter along flow & $0.2 \%$ & $80 \%$ \\
RC with splitter perpendicular flow & $0.75 \%$ & $25 \%$ \\
\hline
\end{tabular}




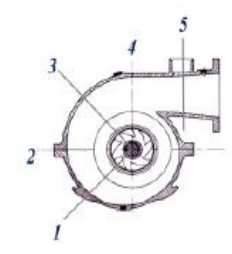

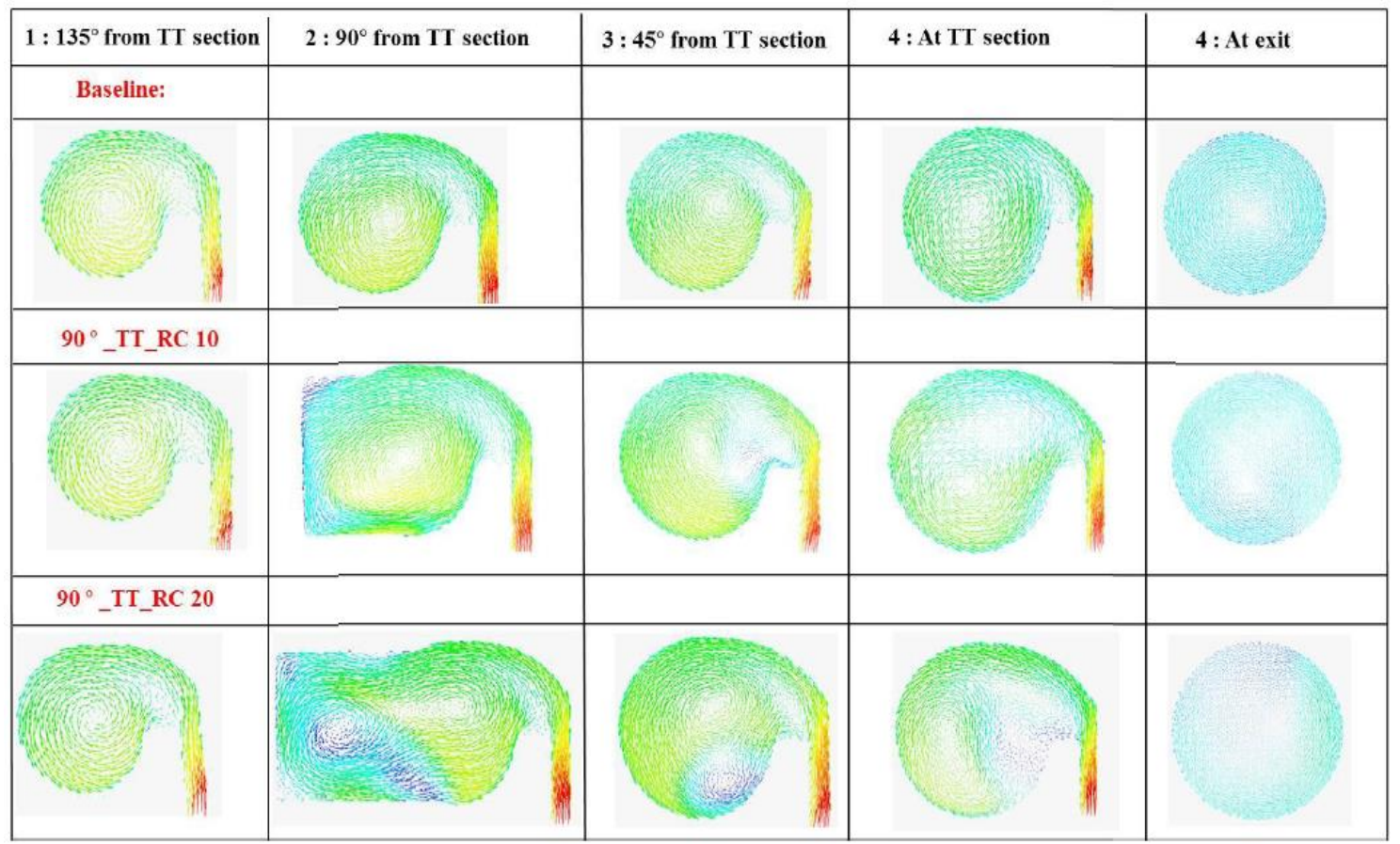

Figure 8. Velocity vector plots showing the evolution of flow at different planes.

\section{RC Without Splitters}

The results for RC modelled on the pipe without the splitter and its comparison of efficiency values with the baseline are shown in Figure 9. It can be observed that a maximum loss of about $1 \%$ is observed near the surge region. The loss at peak was observed to be around $0.5 \%$. Streamlines were plotted to visualise the interaction of primary flow with the RC. It can be observed from Figure 10 that the primary flow interacts with the RC forming a secondary flow in the RC. To further analyse the effect of RC, axial velocity contour was plotted close to the wheel inlet. Loss in momentum of air flow was observed in the case where the $\mathrm{RC}$ was present. The comparison of axial velocity plot in the baseline and pipe with RC is shown in Figure 11. Hence it can be concluded that the RC acts as a disturbance to the inlet flow causing distortion resulting in a drop in compressor efficiency. To further analyse the flow structure, distortion in axial velocity ( $\mathrm{Va}$ ) and relative tangential velocity was plotted on a line close to the shroud ( $80 \%$ span) and close to the wheel. A fast Fourier transform (FFT) was done to filter the blade passing frequency. Inverse FFT was further done to bring back the plot in the time domain. The plot is shown in Figure 12. It can be observed that, there is no significant change in axial velocity but a huge distortion in tangential velocity is observed which reduces the compressor performance. 


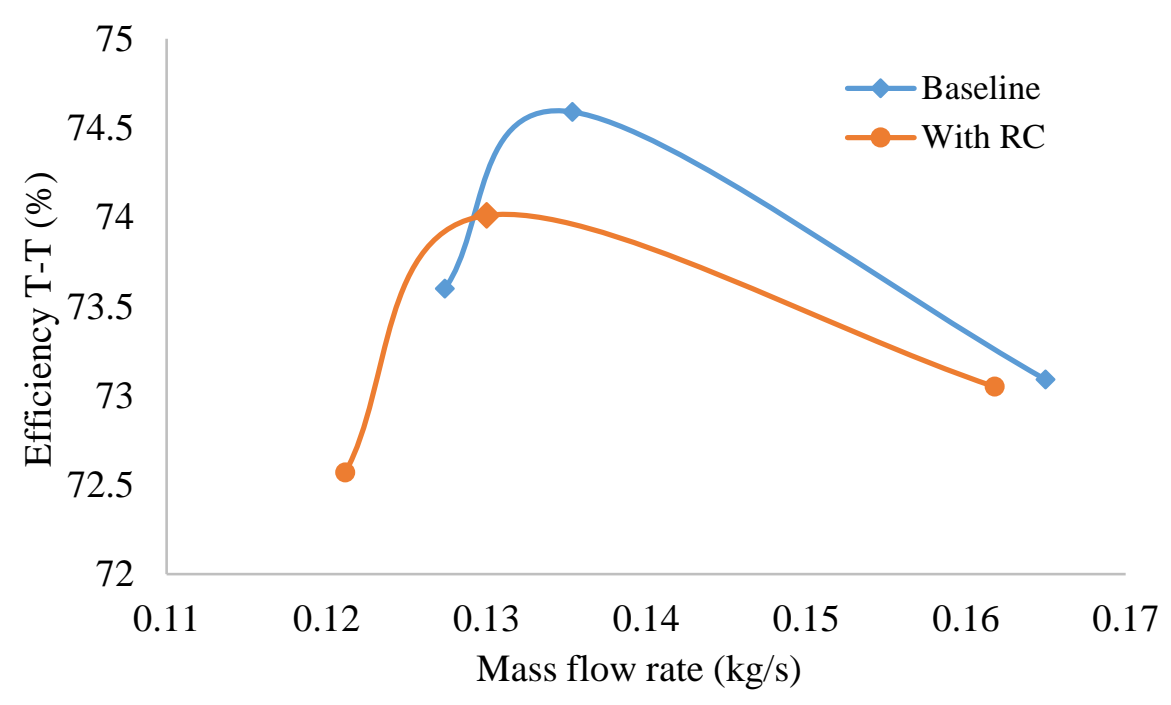

Figure 9. Efficiency curves for compressor with and without RC.
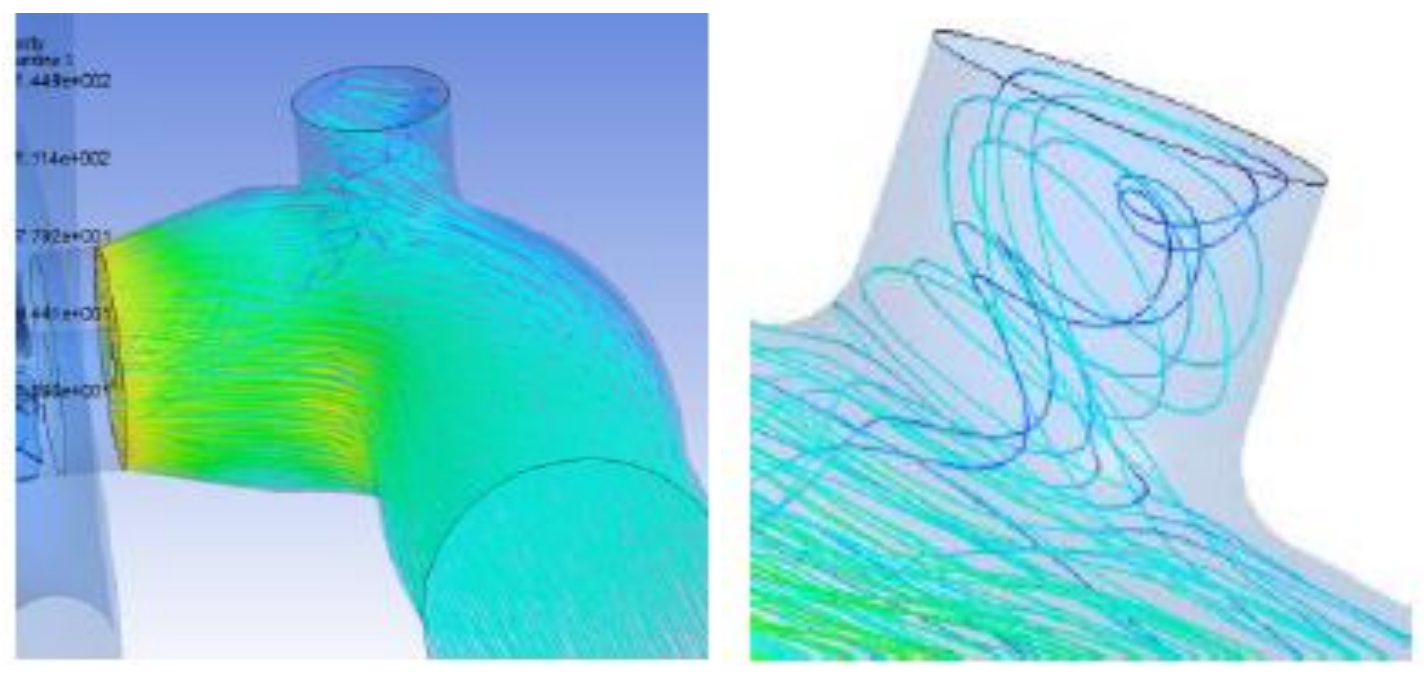

Figure 10. Interaction of primary flow with the RC.
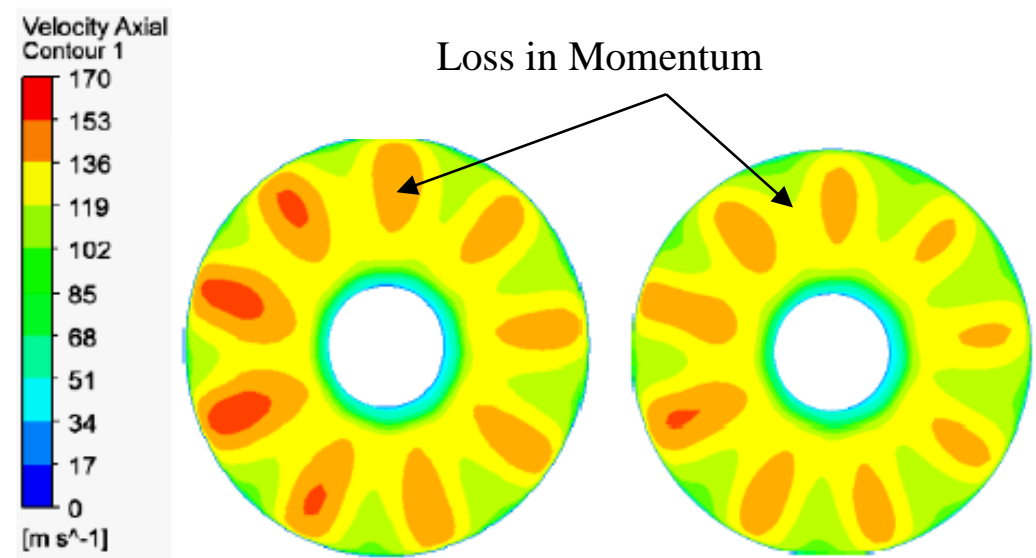

(a) Baseline (No RC)

(b) with RC

Figure 11. Comparison of axial velocity plots for (a) baseline case and (b) compressor with RC. 


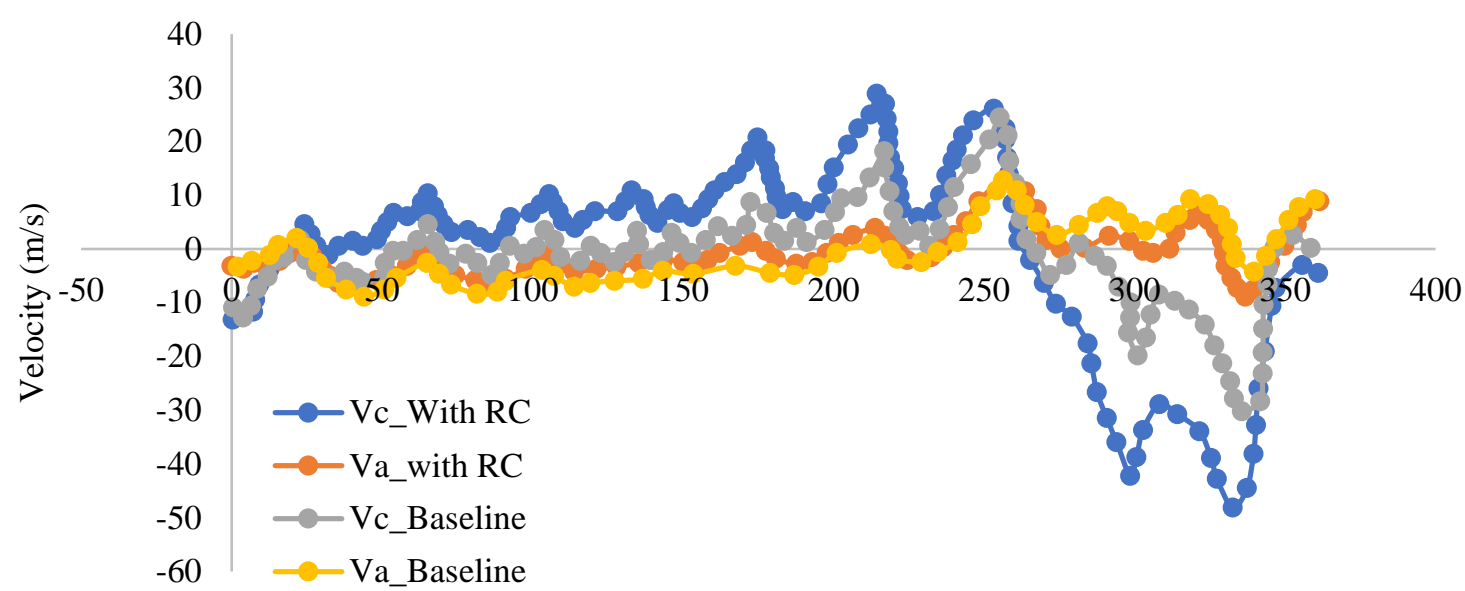

Theta $\left(^{\circ}\right)$

Figure 12. Axial and tangential velocity at inlet for $\mathrm{RC}$ without splitter and baseline.

Further analysis was done to break down the stage loss. Losses were calculated individually for pipe and wheel-volute loss using the same method as that used for the stage. It was observed that, at peak, where the overall stage loss was $0.44 \%$, the pipe loss was $0.39 \%$ whereas the wheel-volute loss was $0.05 \%$ as shown in Figure 13. Hence, maximum effect of $\mathrm{RC}$ was observed in the wheel and volute which resulted from the distortions created in the pipe with RC attached.

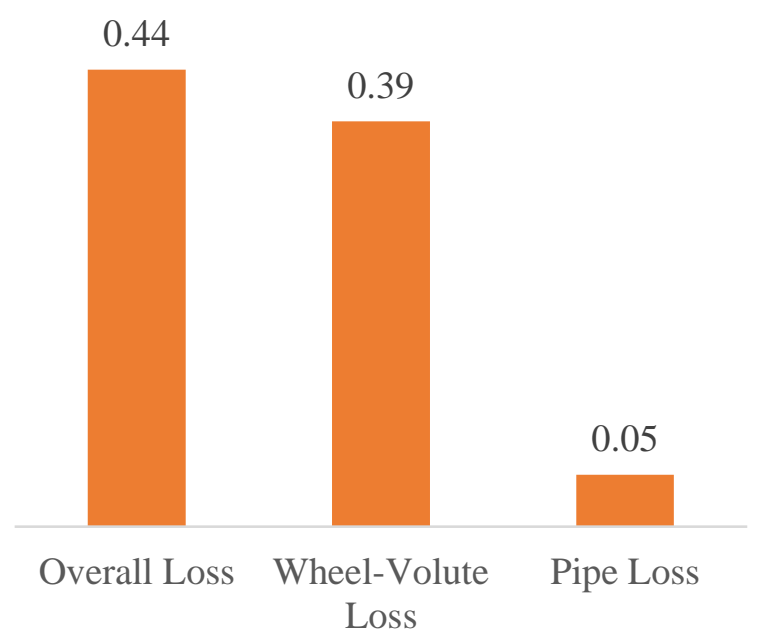

Figure 13. Loss breakout at peak point.

\section{RC with Splitters Along Flow}

The RC was modelled with splitter along the flow. The dimension of the splitter is arbitrarily taken as $2 \mathrm{~mm}$. The RC with splitter modelled along the direction of flow is as shown in Figure 13. 


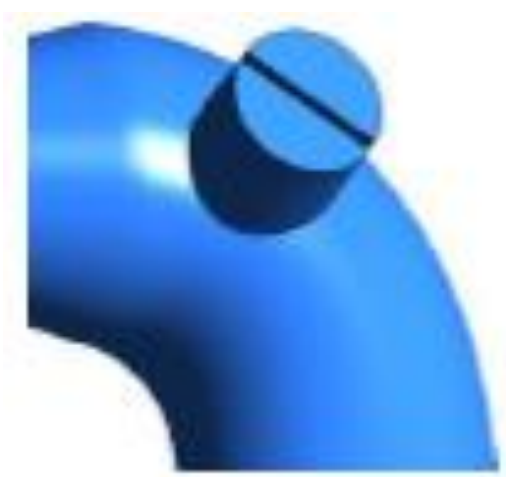

Figure 14. Splitter modelled on RC along the direction of flow.

The efficiency graphs in Figure 15 show that the reduction in compressor efficiency has been significantly reduced due to the presence of the splitter in the RC. Only $0.2 \%$ loss in compressor efficiency is observed at the surge when the splitter is used in a RC which previously showed $1 \%$ loss in compressor efficiency without the splitter. Due to this configuration, the splitter effectively reduces the interference area in the direction normal to the flow which in turn reduces the interaction of the primary flow with the RC. This is shown in the streamline plot in Figure 16. Due to minimal interaction of primary flow with the $\mathrm{RC}$, the compressor performance was not affected. The comparison of axial velocity plots at the wheel inlet is shown in Figure 17. The loss in momentum has been reduced significantly when the splitter was used.

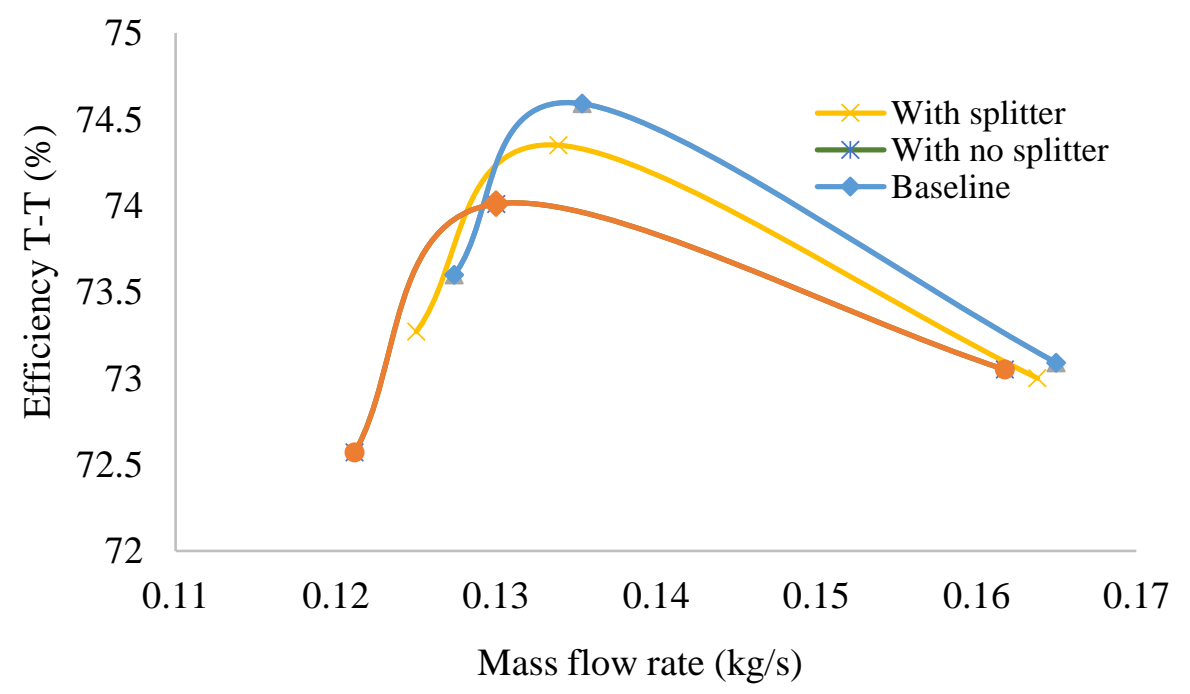

Figure 15. Comparison of efficiency for baseline, with RC and with splitter along flow. 


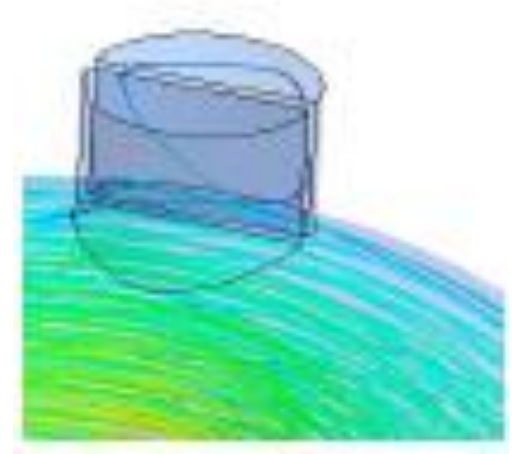

Figure 16. Streamline flow showing feeble interaction of primary flow with RC.

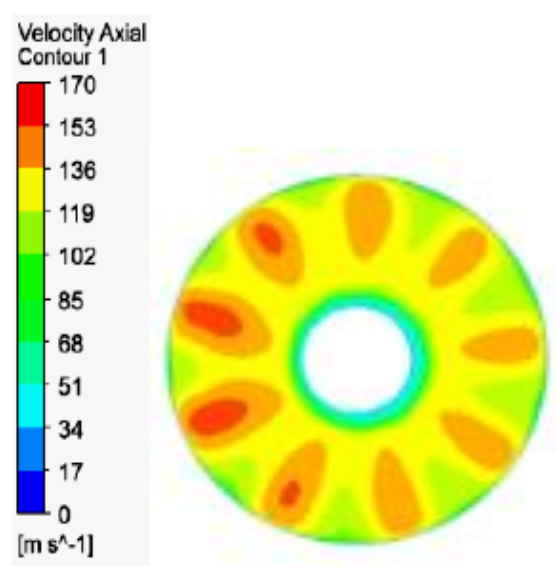

(a) baseline (without RC)

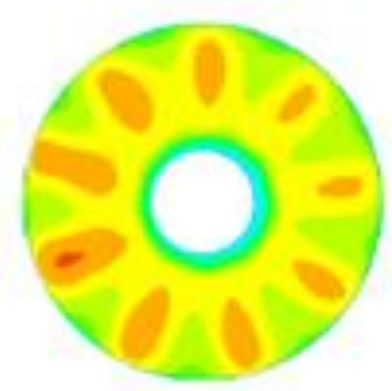

(b) with RC

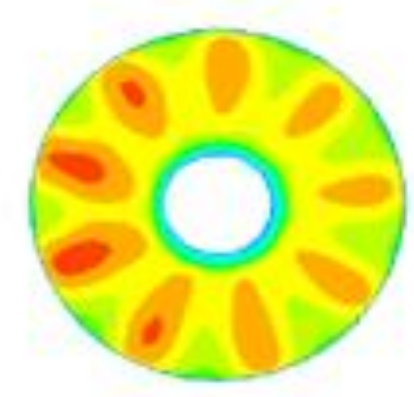

(c) with RC (with splitter)

Figure 17. Comparison of axial velocities at inlet against RC without splitters and with splitters along flow.

\section{RC with Splitters Perpendicular to Flow}

The following Figure 18 represents the splitters used in RC with its orientation perpendicular to the flow. Efficiency curves in Figure 19 show no significant recovery of losses. Drop in compressor efficiency is still prevalent. It can be seen from the streamline plot in Figure 20 that splitters perpendicular to flow direction are not effective in suppressing the vortices and recovering the losses. The reason being that higher frontal area interferes with the primary flow. When the splitter was aligned along the flow, the effective interference area normal to the flow was less, where as in other case half of the circle cavity will directly interfere with the flow. This is illustrated in Figure 21. The comparison of axial velocity plots at the wheel inlet is shown in Figure 22. It can be observed that loss in momentum is not recovered with the use of splitters perpendicular to flow. 


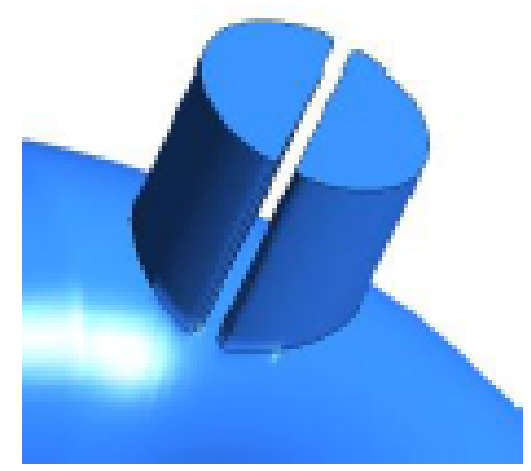

Figure 18. Splitters modelled on RC perpendicular to the flow direction

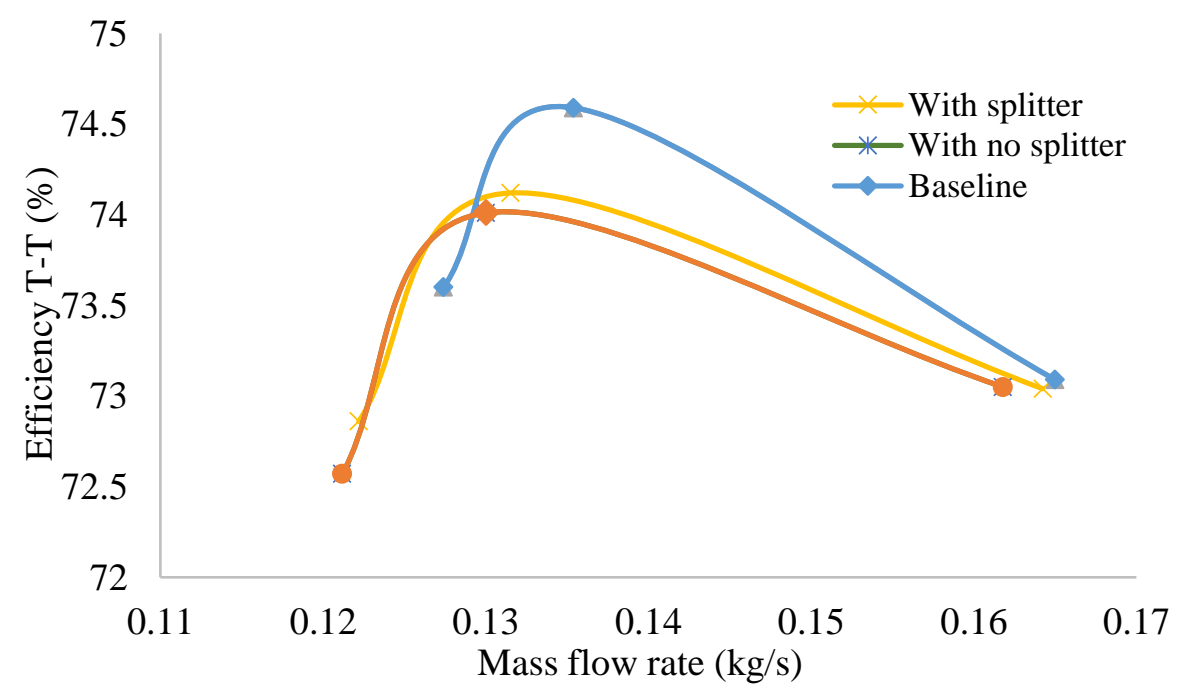

Figure 19. Comparison of efficiency for baseline, with RC and with splitter along the flow.

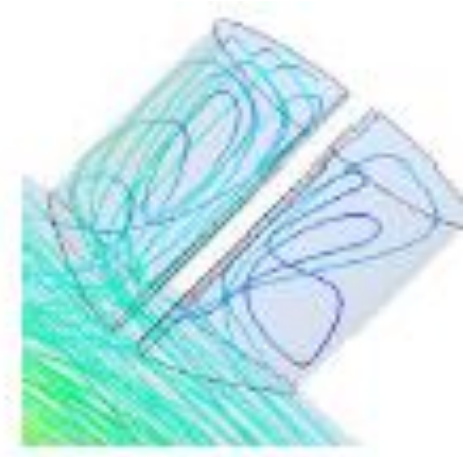

Figure 20. Streamline plot showing strong interaction of primary flow with the RC. 

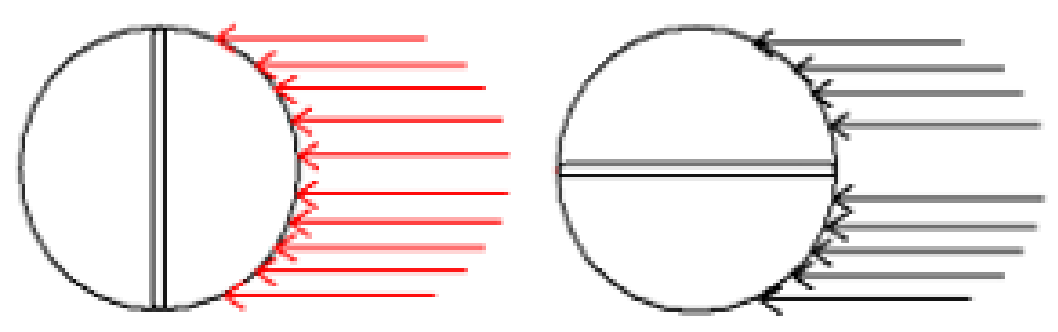

Figure 21. Role of frontal area on flow interaction with RC with splitters in both configurations.

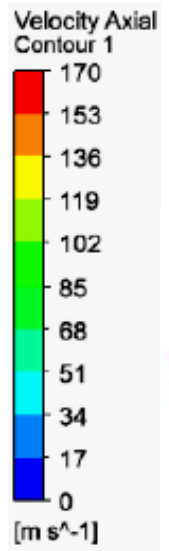

(a) Baseline (No RC)
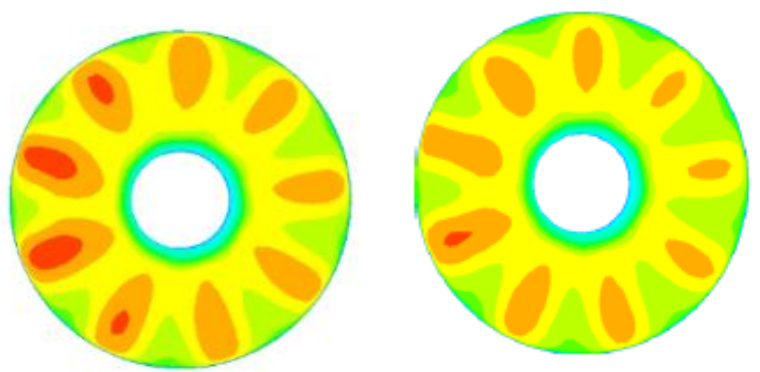

(b) with RC

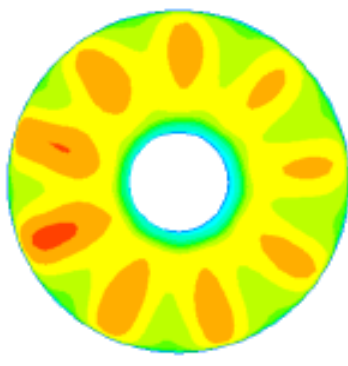

(c) RC (with splitter)

Figure 22. Comparison of axial velocities at inlet against $\mathrm{RC}$ without splitters and with splitters perpendicular to the flow.

The biggest concern associated with the splitter is the obstruction of flow through the valve due to blockage. Due to the presence of the splitter, when the valve is opened, the flow is reduced. But increasing the interference area (size of the RC) and then adding the splitter will solve the above problem. To analyse this, a smaller circular RC of $23 \mathrm{~mm}$ diameter was modelled without splitter. The efficiency graphs as in Figure 23 show that a maximum of $0.4 \%$ drop in efficiency is observed at peak which is worse than a bigger RC with splitter along the flow (only $0.2 \%$ at peak). Hence, a bigger RC can be used in conjunction with a splitter without any noticeable loss in compressor performance. Overall comparison of all cases is shown in Figure 24. It can be observed that RC with splitter along the flow provides maximum restriction to flow interaction with $\mathrm{RC}$ and are hence effective in reducing inlet distortions. 


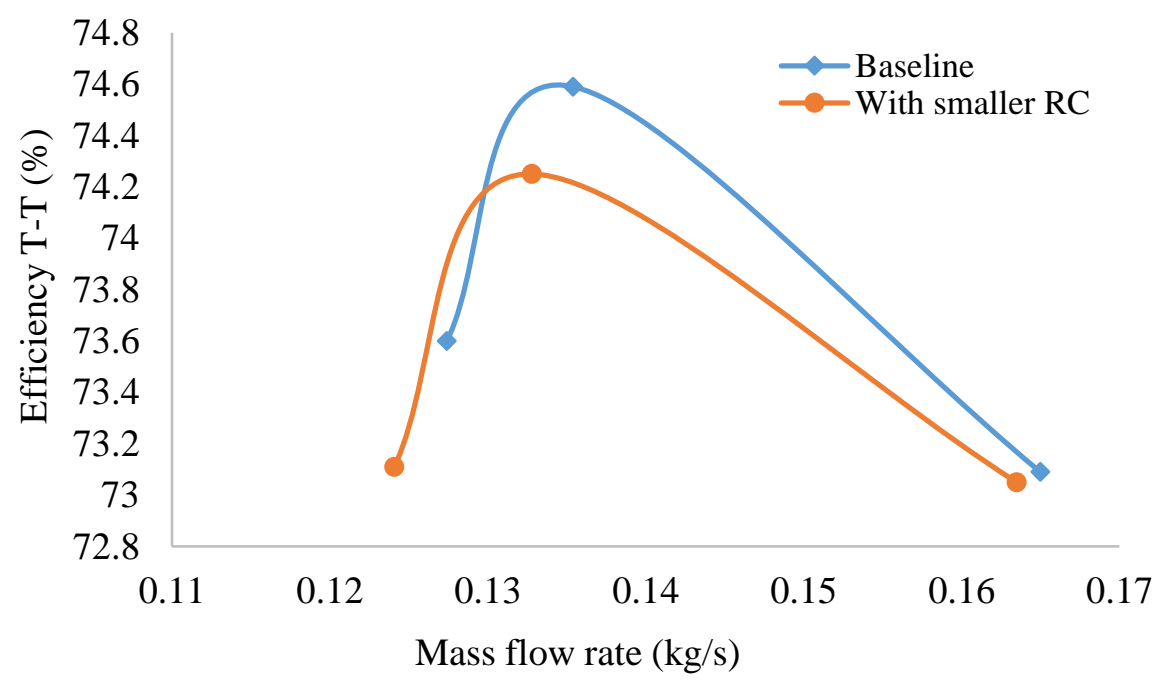

Figure 23. Comparison of efficiency for baseline and smaller RC ( $\varnothing=23 \mathrm{~mm})$.

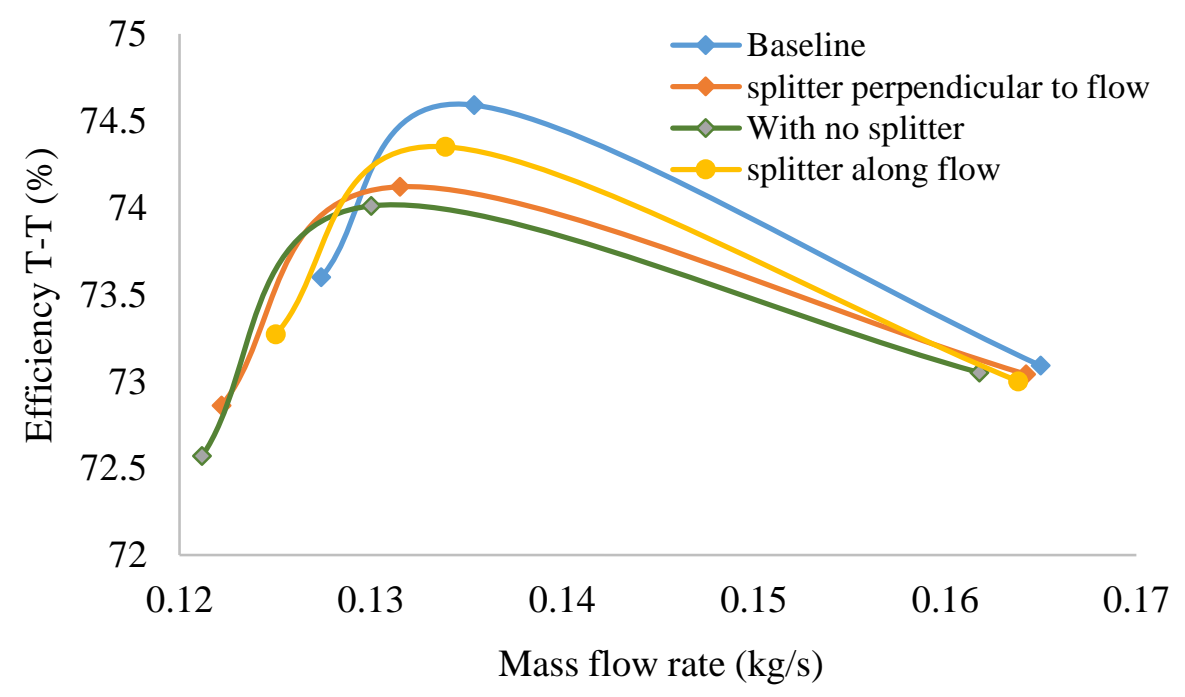

Figure 24. Comparison of all configuration case studies.

\section{CONCLUSION}

Analysis shows that splitters along the flow are effective in reducing compressor losses when a recirculation valve is used. The main reason being that the effective frontal area is split into 2 halves due to which flow interaction with the RC is reduced. Hence, splitters along the flow can be used to restrict entry of fluid through channels placed on bent pipes. The distortion of flow profile is significantly reduced due to which huge drop in compressor efficiency is not observed.

\section{REFERENCES}

[1] Ariga II, Kasai NN, Masuda SS, Watanabe YY, Watanabe II. The effect of inlet distortion on the performance characteristics of a centrifugal compressor. ASME. Journal of Engineering for Power, 1983;105(2):223-230. 
[2] Yang C, Zhou M, Zhu Z, Wang H. Numerical and experimental research on different inlet configurations of high speed centrifugal compressor, Science China Technological Sciences. 2012;55(1): 174-181.

[3] Zemp A, Kammerer A, Abhari RS. Unsteady CFD investigation on inlet distortion in a centrifugal compressor. Turbomachinery, Parts A, B, and C. 2018;6:1909-19.

[4] Roberge JA, Mathisenn PP. Sensitivity analyses to assess the use of CFD to predict the occurrence of vortices near pump intakes. ASME/JSME Joint Fluids Engineering Conference. 1999; FEDSM99-7224.

[5] Cain SA, Padmanabhan M. Numerical simulation of flow distribution and swirl due to a combined pipe bend. In ASME/JSME Joint Fluids Engineering Conference.1999; FEDSM99-7217.

[6] Cheng KC, Lin R, Ou J. Fully developed laminar flow in curved rectangular channels. ASME. Journal of Fluids Engineering. 1976;98(1):41-48.

[7] Abdullah NR, Shahruddin NS, Mamat R, Ihsan Mamat A, Zulkifli A. Effects of air intake pressure on the engine performance, fuel economy and exhaust emissions of a small gasoline engine. Journal of Mechanical Engineering and Sciences. 2014;6:949-58.

[8] Yusop A, Mamat R, Mat Yasin M, Ali OM. Effects of particulate matter emissions of diesel engine using diesel-methanol blends. Journal of Mechanical Engineering and Sciences. 2014; 6:959-67.

[9] Hairuddin AA, Wandel AP, Yusaf T. An introduction to a homogeneous charge compression ignition engine. Journal of Mechanical Engineering and Sciences. 2014;7:1042-52.

[10] Kajishima T, Miyake Y, Inaba T. Numerical simulation of laminar flow in curved ducts of rectangular cross section. JSME International Journal. Ser. 2, Fluids Engineering, Heat Transfer, Power, Combustion, Thermophysical Properties. 1989; 32(4): 516-522.

[11] Sankaralingam RK, Venugopal T. Current trends on combustion control methods using fuel reactivities. International Journal of Automotive and Mechanical Engineering. 2016;13:3788-11.

[12] Kapilan N, Ashok Babu TP, Reddy RP. Improvement of performance of dual fuel engine operated at part load. International Journal of Automotive and Mechanical Engineering. 2010;2:200-10.

[13] Azad AK, Ameer Uddin SM, Alam MM. A comprehensive study of DI diesel engine performance with vegetable oil: an alternative boi-fuel source of energy. International Journal of Automotive and Mechanical Engineering. 2012;5:57686.

[14] Soon LB, M. Rus AZ, Hasan S. Continuous biodiesel production using ultrasound clamp on tubular reactor. International Journal of Automotive and Mechanical Engineering. 2013;8:1396-405.

[15] Yusaf T, Hamawand I, Baker P, Najafi G. The effect of methanol-diesel blended ratio on CI engine performance. International Journal of Automotive and Mechanical Engineering. 2013;8:1385-95.

[16] Mat Yasin MH, Mamat R, Sharma KV, Yusop AF. Influence of palm methyl ester $(\mathrm{PME})$ as an alternative fuel in multicylinder diesel engine. Journal of Mechanical Engineering and Sciences. 2012;3:331-9.

[17] Jye AETS, Pesiridis A, Rajoo S. Effects of mechanical turbo compounding on a turbocharged diesel engine. SAE Technical Paper No. 2013-01-0103; 2013. 
[18] Ghobadian B, Najafi G, Nayebi MA semi-empirical model to predict diesel engine combustion parameters. Journal of Mechanical Engineering and Sciences. 2013; 4:373-82. 